\title{
Original Research \\ Impact of aortic atheroma and distensibility on diastolic function and prognosis in patients with ischemic stroke
}

\author{
Dae-Young Kim ${ }^{1}$, In-Soo Kim², Yo Han Jung ${ }^{3}$, Kyung Yul Lee ${ }^{3}$, Jong-Youn Kim², \\ Pil-Ki Min², Young Won Yoon ${ }^{2}$, Byoung Kwon Lee ${ }^{2}$, Bum-Kee Hong ${ }^{2}$, Se-Joong Rim², \\ Hyuck Moon Kwon ${ }^{2}$, Eui-Young Choi ${ }^{2, *}$
}

\author{
${ }^{1}$ Division of Cardiology, Severance Cardiovascular Hospital, Yonsei University College of Medicine, 03722 Seoul, Republic of Korea \\ ${ }^{2}$ Division of Cardiology, Heart Center, Gangnam Severance Hospital, Yonsei University College of Medicine, 06273 Seoul, Republic of Korea \\ ${ }^{3}$ Department of Neurology, Gangnam Severance Hospital, 06273 Seoul, Republic of Korea \\ *Correspondence: choi0928@yuhs.ac (Eui-Young Choi) \\ Academic Editors: Brian Tomlinson and Takatoshi Kasai \\ Submitted: 25 October 2021 Revised: 30 November 2021 Accepted: 7 December 2021 Published: 11 January 2022
}

\begin{abstract}
Background: Patients with ischemic stroke are vulnerable to heart failure with preserved ejection fraction (HFpEF) because these conditions share common risk factors. Although evaluation of the ascending aorta, aortic arch, and proximal descending thoracic aorta is an essential step to determine the source of the causative embolism, the relationship between the degree of aortic atheroma and left ventricular (LV) diastolic function has not been extensively investigated. Methods: We analyzed the transesophageal and transthoracic echocardiography in ischemic stroke patients. Patients with previous coronary artery disease, valvular heart disease of more than moderate degree, and an LV ejection fraction of less than 50\% were excluded. The relationships between the grade of the aortic atheroma, aortic stiffness indexes, and diastolic functional indexes were evaluated. Results: In 295 patients, the atheroma grade was significantly correlated with aortic stiffness index, ratio of mitral annular and inflow velocities (E/e'), left atrial volume index, and LV diastolic elastance. With further adjustment for age, hypertension, diabetes, estimated glomerular filtration rate, left atrial volume index, and LV mass index, the significance of the atheroma grade was attenuated. In the subgroup analysis, the atheroma grade was significantly and independently related to $\mathrm{E} / \mathrm{e}$ ' in women $(\beta=0.181, p=0.032)$, but not in men. However, atheroma grade was not associated with poor clinical outcomes in either sex. Conclusions: Aortic atheroma grade was significantly and independently related to LV diastolic function, especially in women. This suggests that aortic atheroma is an index of arterial stiffness and a potential risk factor for HFpEF through ventricular-vascular interactions, especially in women.
\end{abstract}

Keywords: Ischemic stroke; Aortic atheroma; Diastolic function; Prognosis

\section{Introduction}

Patients who undergo ischemic stroke are vulnerable to heart failure with preserved ejection fraction (HFpEF) because they have common risk factors, such as hypertension, diabetes, arrhythmia, dyslipidemia, old age, and obesity [1]. These two diseases have the same pathophysiological pathway. Arterial stiffness is the most important component of increased systolic and pulse pressure and is a major cause of diverse cardiovascular complications [2]. It can also be a possible risk factor for both atherosclerosis and heart failure with diastolic dysfunction, and thoracic aortic atherosclerosis is considered an intensive risk factor for embolic stroke $[3,4]$.

Evaluation of the ascending aorta (AA), aortic arch, and proximal descending thoracic aorta (pDTA) using transesophageal echocardiography (TEE) is an essential test for determining the presence of embolism in patients with ischemic stroke [5,6], and the degree of aortic atheroma is known to be related to increased aortic stiffness and decreased aortic distensibility [7]. Although a significant re- lationship has been recognized between aortic stiffness and left ventricular (LV) diastolic function, the connection between the degree of aortic atheroma and LV diastolic function has not been extensively investigated. Previous studies showed that there were significant sex differences in cause, presentation and prognosis of ischemic stroke [8]. In addition, women are more vulnerable to HFpEF though impaired ventricular-arterial coupling $[9,10]$. Therefore, it needs to reveal any sex differences in contributors to prognosis in patients with ischemic stroke.

The aim of this article was to examine the correlation between the severity of aortic atheroma as an indicator of aortic stiffness and parameters that imply LV diastolic dysfunction in a group of patients who were diagnosed with ischemic stroke. Since it is known that there are sex differences in the association between arterial stiffness and LV diastolic function [9], we also examined whether gender differences exist in the relation between aortic atheroma and diastolic dysfunction. The mid-term prognostic value of aortic atheroma and stiffness was also evaluated. 


\section{Method}

\subsection{Study population}

We collected consecutive data of patients treated from January 2006 to May 2019 in a tertiary hospital, who were diagnosed with ischemic stroke or transient ischemic attack (TIA) and underwent transthoracic echocardiography (TTE) and TEE simultaneously with the aim of identifying the focus of embolic stroke or TIA Ischemic stroke or TIA was diagnosed based on the presence of neurologic deficits and findings obtained using brain computed tomography or magnetic resonance imaging. Neurologic deficits were identified by an expert in the neurology department at the time of diagnosis in the hospital. Among the patients with ischemic stroke or TIA, only those who met the following criteria performed TEE as follows: (1) relatively young age as under 65 , (2) age $\geq 65$ years who had recurrent events of ischemic stroke or TIA, (3) multiple embolic strokes, (4) suspicious of embolic cause without the evidence of other definite etiology by the clinician's decision, or (5) any history of cancer. Therefore, among total of 4206 patients with ischemic stroke or TIA in this period, after excluding the patients who did not meet the criteria specified above, denied to undergo TEE, or with poor systemic conditions unable to tolerate TEE. 442 patients underwent TEE examination. We excluded patients who had previous acute coronary syndrome or coronary artery disease at the left main coronary artery or at least two coronary arteries that were revealed as having $50 \%$ or more narrowing on coronary angiography, had valvular heart disease more than moderate degree on TTE or TEE, had heart failure with reduced ejection fraction less than $50 \%$, had not been able to have diastolic parameters measured as E/e' on TTE, and had been diagnosed with hypertrophic cardiomyopathy, restrictive cardiomyopathy, or constrictive cardiomyopathy. After excluding the patients according to the criteria specified above, a total of 295 patients were finally enrolled in this study. We retrospectively reviewed the patients' medical records, clinical characteristics, medications, echocardiographic imaging data, and clinical outcomes.

\subsection{Collection of clinical outcomes data}

After discharge from the hospital, the patients were scheduled to visit the outpatient clinic regularly for at least 3 months. The primary endpoint was a composite of hospitalization due to heart failure, acute myocardial infarction or acute coronary syndrome, ischemic stroke or TIA, and death from any cause. Heart failure hospitalization was defined based on symptoms of dyspnea of at least New York Heart Association Class III, as well as the need for diuretics or vasodilators, and evidence of pulmonary edema or pleural effusion on chest radiograph. If a patient had more than one clinical outcome, the first event was included in the primary outcome analysis.

\subsection{Echocardiography}

Standard 2-dimensional and Doppler measurements were conducted based on recommended guidelines [11]. LV ejection fraction, left atrial (LA) volume index $\left(\mathrm{mL} / \mathrm{m}^{2}\right)$, and LV mass index $\left(\mathrm{g} / \mathrm{m}^{2}\right)$ were measured with the apical two- and four-chamber variants using the biplane Simpson's method. Diastolic parameters such as peak E velocity of early diastolic mitral inflow and early velocity in the diastolic mitral annulus of the septum (e') were measured using pulse-wave and tissue Doppler. The LA volume index and E/e', which might be a good parameter for estimating LV filling pressure, were used as indexes of diastolic dysfunction [12]. LV diastolic elastance was calculated using the ratio of E/e' over the stroke volume and used as a diastolic functional index [13]. Among the echocardiographic parameters, presence of dense mitral annular calcification (MAC) was known to be related with complex aortic atherosclerosis [14]. To determine the relationship between those markers, we also reviewed whether there was dense MAC which was defined as the maximal thickness of the calcification at the base of the mitral leaflets over $5 \mathrm{~mm}$ in echocardiography.

\subsection{Measurement of the grade of atheroma and aortic stiffness}

On TEE examination, the location and grade of the aortic atheroma were measured using the guidelines provided by the American Society of Echocardiography [15]. Grade 1, intimal thickness $<2 \mathrm{~mm} ; 2$, intimal thickening of 2-3 mm; 3, atheroma $>3-5 \mathrm{~mm}$ (no mobile/ulcerated components); 4 , atheroma $>5 \mathrm{~mm}$ (no mobile/ulcerated components), and 5 as grade 2, 3, or 4 atheroma plus mobile or ulcerated components. The separation of the location of the ascending aorta (AA), aortic arch, and proximal descending thoracic aorta (pDTA) was performed by defining the aortic arch as the portion from the end of the ascending aorta to the origin of the left subclavian artery. We used the TEE image at the level of pDTA to calculate the aortic stiffness index $(\beta)$. From this image, the maximum diameter of the aortic lumen during the ejection period $\left(\mathrm{D}_{\max }\right)$ and minimum diameter of the aortic lumen during the pre-ejection period $\left(\mathrm{D}_{\min }\right)$ were measured. Next, the aortic stiffness index was calculated as follows: aortic stiffness index $(\beta)=\ln$ (systolic blood pressure / diastolic blood pressure) / [( $\mathrm{D}_{\max }$ $\left.\left.-\mathrm{D}_{\text {min }}\right) / \mathrm{D}_{\min }\right]$, where $\ln$ indicates the natural logarithm [6]. Aortic distensibility was computed using the following formula: aortic distensibility $=\left(\mathrm{D}_{\max }-\mathrm{D}_{\min }\right) /\left(\mathrm{D}_{\min } \times\right.$ pulse pressure) [16]. Effective arterial elastance was estimated by calculating the ratio of end-systolic pressure to stroke volume [17]. Total arterial compliance was computed as the stroke volume/pulse pressure.

\subsection{Statistical analysis}

Continuous variables are expressed as mean \pm standard deviation, and categorical variables are presented as 
Table 1. Baseline characteristics.

\begin{tabular}{|c|c|c|c|c|}
\hline & Total & Men & Women & \multirow{2}{*}{$p$ value } \\
\hline & $(\mathrm{n}=295)$ & $(\mathrm{n}=209)$ & $(\mathrm{n}=86)$ & \\
\hline Ischemic stroke, n (\%) & $283(95.9)$ & $201(96.2)$ & $82(95.3)$ & 0.745 \\
\hline Transient ischemic attack, $\mathrm{n}(\%)$ & $12(4.1)$ & $8(3.8)$ & $4(4.7)$ & \\
\hline Age, years & $58.0 \pm 14.1$ & $57.3 \pm 13.6$ & $59.9 \pm 15.4$ & 0.154 \\
\hline Body surface area, $\mathrm{m}^{2}$ & $1.77 \pm 0.2$ & $1.84 \pm 0.2$ & $1.59 \pm 0.1$ & $<0.001$ \\
\hline Systolic blood pressure, $\mathrm{mmHg}$ & $134.2 \pm 21.5$ & $134.5 \pm 20.6$ & $133.3 \pm 23.7$ & 0.655 \\
\hline Diastolic blood pressure, $\mathrm{mmHg}$ & $81.6 \pm 14.5$ & $82.7 \pm 14.6$ & $79.0 \pm 14.0$ & 0.049 \\
\hline Pulse pressure, $\mathrm{mmHg}$ & $52.6 \pm 14.8$ & $51.9 \pm 13.0$ & $54.3 \pm 18.4$ & 0.271 \\
\hline Hypertension, n (\%) & $168(56.9)$ & $121(57.9)$ & $47(54.7)$ & 0.609 \\
\hline Diabetes mellitus, n (\%) & $66(22.4)$ & $49(23.4)$ & $17(19.8)$ & 0.491 \\
\hline Dyslipidemia, n (\%) & $128(43.4)$ & $95(45.5)$ & $33(38.4)$ & 0.265 \\
\hline Atrial fibrillation, n (\%) & $44(14.9)$ & $35(16.7)$ & $9(10.5)$ & 0.169 \\
\hline Previous MI, n (\%) & $7(2.4)$ & $4(1.9)$ & $3(3.5)$ & 0.419 \\
\hline Previous stroke, n (\%) & $37(12.5)$ & $23(11.0)$ & $14(16.3)$ & 0.214 \\
\hline Smoking, n (\%) & $91(30.8)$ & $76(36.4)$ & $15(17.4)$ & 0.001 \\
\hline \multicolumn{5}{|l|}{ Medications, n (\%) } \\
\hline RAAS blockers & $106(35.9)$ & $78(37.3)$ & $28(32.6)$ & 0.438 \\
\hline Beta-blockers & $46(15.6)$ & $40(19.1)$ & $6(7.0)$ & 0.009 \\
\hline $\mathrm{CCB}$ & $93(31.5)$ & $63(30.1)$ & $30(34.9)$ & 0.426 \\
\hline Diuretics & $40(13.6)$ & $28(13.4)$ & $12(14.0)$ & 0.899 \\
\hline Statin & $245(83.1)$ & $177(84.7)$ & $68(79.1)$ & 0.242 \\
\hline Antiplatelet agents & $254(86.1)$ & $180(86.1)$ & $74(86.0)$ & 0.986 \\
\hline Anticoagulants & $81(27.5)$ & $60(28.7)$ & $21(24.4)$ & 0.453 \\
\hline \multicolumn{5}{|l|}{ Laboratory data } \\
\hline Hemoglobin, g/dL & $14.2 \pm 2.1$ & $14.7 \pm 1.9$ & $12.8 \pm 1.9$ & $<0.001$ \\
\hline Hematocrit, \% & $41.8 \pm 6.0$ & $43.3 \pm 5.5$ & $38.3 \pm 5.6$ & $<0.001$ \\
\hline $\mathrm{eGFR}, \mathrm{mL} / \mathrm{min}$ & $92.7 \pm 17.9$ & $92.4 \pm 17.0$ & $93.4 \pm 20.0$ & 0.657 \\
\hline Total cholesterol, mg/dL & $174.3 \pm 42.6$ & $174.6 \pm 42.3$ & $173.5 \pm 43.6$ & 0.844 \\
\hline Brain lesion & & & & 0.686 \\
\hline Left side, $\mathrm{n}(\%)$ & $111(37.6)$ & $82(39.2)$ & $29(33.7)$ & \\
\hline Right side, n (\%) & $108(36.6)$ & $72(34.4)$ & $36(41.9)$ & \\
\hline Bilateral, n (\%) & $41(13.9)$ & $28(13.4)$ & $13(15.1)$ & \\
\hline Cerebellum, n (\%) & $16(5.4)$ & $12(5.7)$ & $4(4.7)$ & \\
\hline Undefined, n (\%) & $19(6.4)$ & $15(7.2)$ & $4(4.7)$ & \\
\hline
\end{tabular}

MI, myocardial infarction; RAAS, renin-angiotensin-aldosterone system; CCB, calcium channel blocker; eGFR, estimated glomerular filtration rate.

counts and percentages. The chi-squared test and independent $t$-test were used to compare categorical and continuous variables. One-way analysis of variance (ANOVA) and post-hoc analysis (HSD and Bonferroni correction) were used to compare the severity of aortic atheroma and were classified into grades from 1 to 5 . The relationships between the independent variables and the severity of diastolic functional impairment were determined using univariate and multivariate linear regression analyses. Clinical outcomes were assessed using Kaplan-Meier analysis, and the predictors of clinical outcomes were evaluated using Cox regression analysis. Statistical significance was set at $p<0.05$. Data were analyzed using Statistical Package for the Social Sciences version 25.0 (IBM Corporation, Armonk, NY, USA).

\section{Results}

\subsection{Baseline clinical and echocardiographic characteristics}

Of the 295 patients (mean age, $58.0 \pm 14.1$ years), 209 (70.8\%) were male. Among the 295 patients, 283 (95.9\%) underwent ischemic stroke and the remainder 12 underwent transient ischemic attack (4.1\%). Brain lesions were located on the left side in 111 patients (37.6\%), on the right 
Table 2. Baseline echocardiographic parameters.

\begin{tabular}{|c|c|c|c|c|}
\hline & Total & Male & Female & \multirow{2}{*}{$p$ value } \\
\hline & $(\mathrm{n}=295)$ & $(\mathrm{n}=209)$ & $(\mathrm{n}=86)$ & \\
\hline \multicolumn{5}{|l|}{ TEE findings } \\
\hline Presence of visible atheromatous plaques, $n(\%)$ & $132(44.7)$ & $99(47.4)$ & $33(38.4)$ & 0.158 \\
\hline Atheroma grade & & & & 0.654 \\
\hline 1 & $163(55.3)$ & $110(52.6)$ & $53(61.6)$ & \\
\hline 2 & $52(17.6)$ & $38(18.2)$ & $14(16.3)$ & \\
\hline 3 & $41(13.9)$ & $30(14.4)$ & $11(12.8)$ & \\
\hline 4 & $33(11.2)$ & $26(12.4)$ & $7(8.1)$ & \\
\hline 5 & $6(2.0)$ & $5(2.4)$ & $1(1.2)$ & \\
\hline Indexed annulus diameter, $\mathrm{mm} / \mathrm{m}^{2}$ & $11.4 \pm 1.6$ & $11.3 \pm 1.5$ & $11.8 \pm 1.6$ & 0.011 \\
\hline Indexed SOV diameter, $\mathrm{mm} / \mathrm{m}^{2}$ & $18.8 \pm 2.6$ & $18.5 \pm 2.5$ & $19.6 \pm 2.7$ & 0.001 \\
\hline Indexed STJ diameter, $\mathrm{mm} / \mathrm{m}^{2}$ & $15.3 \pm 2.2$ & $15.0 \pm 2.1$ & $15.9 \pm 2.3$ & 0.001 \\
\hline Indexed AA diameter, $\mathrm{mm} / \mathrm{m}^{2}$ & $18.7 \pm 3.0$ & $18.2 \pm 2.5$ & $20.0 \pm 3.4$ & $<0.001$ \\
\hline Indexed pDTA-D $\mathrm{D}_{\max }, \mathrm{mm} / \mathrm{m}^{2}$ & $14.6 \pm 2.3$ & $14.4 \pm 2.2$ & $14.9 \pm 2.6$ & 0.081 \\
\hline Indexed pDTA-D $\min , \mathrm{mm} / \mathrm{m}^{2}$ & $13.8 \pm 2.4$ & $13.7 \pm 2.3$ & $14.1 \pm 2.6$ & 0.183 \\
\hline \multicolumn{5}{|l|}{ TTE findings } \\
\hline LV ejection fraction, $\%$ & $64.9 \pm 6.1$ & $64.4 \pm 5.9$ & $65.9 \pm 6.5$ & 0.050 \\
\hline LA volume index, $\mathrm{mL} / \mathrm{m}^{2}$ & $30.3 \pm 12.9$ & $31.1 \pm 13.4$ & $28.2 \pm 11.3$ & 0.085 \\
\hline $\mathrm{LV}$ mass index, $\mathrm{g} / \mathrm{mL}$ & $90.0 \pm 20.2$ & $92.4 \pm 20.2$ & $84.2 \pm 19.1$ & 0.001 \\
\hline $\mathrm{E}, \mathrm{m} / \mathrm{s}$ & $0.74 \pm 0.19$ & $0.74 \pm 0.20$ & $0.73 \pm 0.18$ & 0.746 \\
\hline $\mathrm{e}^{\prime}, \mathrm{m} / \mathrm{s}$ & $0.07 \pm 0.02$ & $0.07 \pm 0.02$ & $0.07 \pm 0.03$ & 0.034 \\
\hline $\mathrm{E} / \mathrm{e}^{\prime}$ & $10.8 \pm 3.7$ & $10.4 \pm 3.5$ & $11.8 \pm 3.9$ & 0.003 \\
\hline $\mathrm{LV}$ diastolic elastance, $1 / \mathrm{mL}$ & $0.17 \pm 0.08$ & $0.16 \pm 0.07$ & $0.20 \pm 0.10$ & $<0.001$ \\
\hline Dense mitral annular calcification, $\mathrm{n}(\%)$ & $26(8.8)$ & $12(5.7)$ & $14(16.3)$ & 0.004 \\
\hline Aortic distensibility, $\mathrm{mm} / \mathrm{mmHg}$ & $0.12 \pm 0.06$ & $0.12 \pm 0.06$ & $0.13 \pm 0.07$ & 0.094 \\
\hline Aortic stiffness index, $\mathrm{kPa}$ & $11.3 \pm 7.8$ & $11.5 \pm 7.7$ & $11.0 \pm 7.9$ & 0.601 \\
\hline Effective arterial elastance, $\mathrm{mmHg} / \mathrm{mL}$ & $1.84 \pm 0.54$ & $1.78 \pm 0.50$ & $1.99 \pm 0.60$ & 0.002 \\
\hline Total arterial compliance, $\mathrm{mL} / \mathrm{mmHg}$ & $1.38 \pm 0.53$ & $1.41 \pm 0.44$ & $1.27 \pm 0.68$ & 0.033 \\
\hline \multicolumn{5}{|c|}{ TTE, transthoracic echocardiography; TEE, transesophageal echocardiography; SOV, sinus of } \\
\hline \multicolumn{5}{|c|}{$\begin{array}{l}\text { Valsalva; STJ, sinotubular junction; AA, ascending aorta; pDTA, proximal descending thoracic } \\
\text { aorta; LV, left ventricular; LA, left atrial; E, early diastolic mitral inflow velocity; e', early }\end{array}$} \\
\hline
\end{tabular}

side in $108(36.6 \%)$, bilaterally in $41(13.9 \%)$, and in the cerebellum in $16(5.4 \%)$ patients; there were no significant differences between the sexes. On TTE findings, women had lower LV mass index $(84.2 \pm 19.1 \mathrm{~g} / \mathrm{mL}$ vs. 92.4 $\pm 20.2 \mathrm{~g} / \mathrm{mL}, p=0.001)$ but higher E/e' $(11.8 \pm 3.9 \mathrm{vs}$. $10.4 \pm 3.5, p=0.003)$ and LV diastolic elastance $(0.20$ \pm 0.10 vs. $0.16 \pm 0.07, p<0.001)$. Patients with dense MAC were $26(8.8 \%)$ of all study population and women had more patients with dense MAC $(16.3 \%$ vs. $5.7 \%, p=$ 0.004). Although, the prevalence of MAC was higher in aortic atheroma $(+)$ group but it did not reach the statistical significance in our study $(11.4 \%$ vs. $6.7 \%, p=0.164)$ (Supplementary Table 1). The baseline characteristics and echocardiographic findings of the two groups are described in Tables 1,2 .
3.2 Sex differences in degree of aortic atheroma and aortic stiffness indexes

A total of 132 patients (44.7\%) had visible aortic atheromatous plaques. Among them, the grade of atheroma had a similar distribution, except for grade 5. Fifty-eight patients (44\%) had aortic atheroma only at the aortic arch, 40 patients $(30 \%)$ at both the aortic arch and pDTA, and 28 patients $(21 \%)$ had aortic atheroma only at the pDTA. Six patients $(5 \%)$ had aortic atheroma in the lesion, including the ascending aorta (Fig. 1). There was no difference in the distribution of atheroma grades between both sexes. The female group had higher dimensions of the aortic root when indexed according to body surface area. Women had higher effective arterial elastance $(1.99 \pm 0.60 \mathrm{mmHg} / \mathrm{mL}$ vs. $1.78 \pm 0.50 \mathrm{mmHg} / \mathrm{mL}, p=0.002)$ and lower total arterial compliance $(1.27 \pm 0.68 \mathrm{~mL} / \mathrm{mmHg}$ vs. $1.41 \pm 0.44$ 


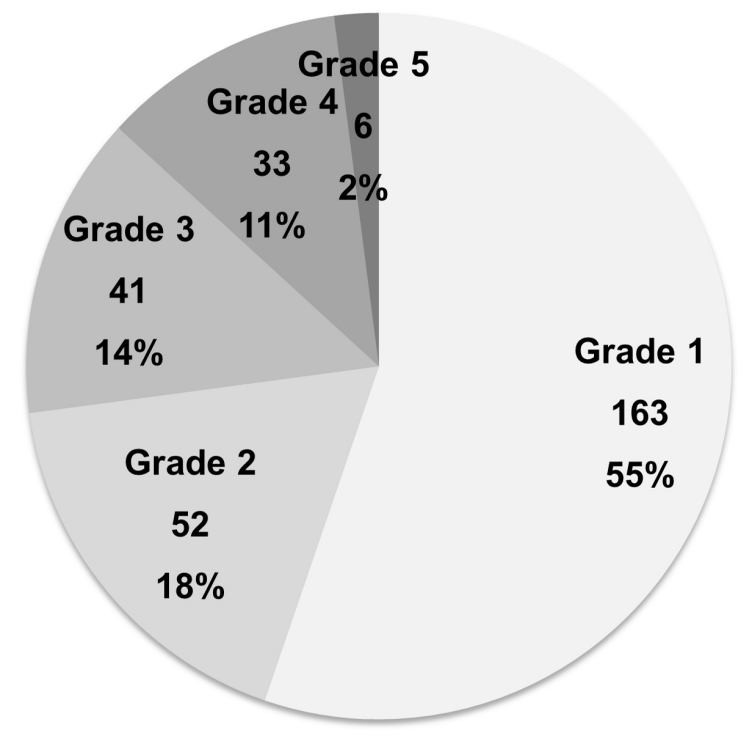

Grade 1 Grade 2 Grade 3 Grade 4 arade 5
- Location

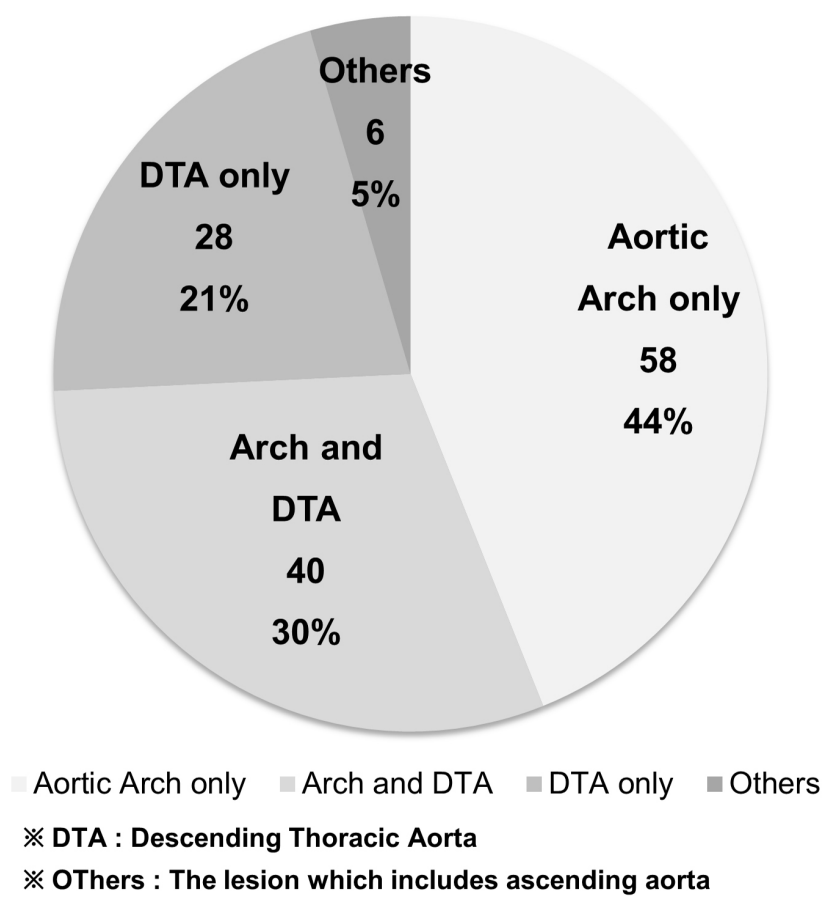

Fig. 1. Grade distribution of aortic atheroma and location of ischemic stroke. More than $50 \%$ of the patients are classified as grade 1 (163 patients, 55\%). Fifty-eight patients (44\%) have aortic atheroma only at the aortic arch, 40 patients (30\%) at both the aortic arch and DTA, and 28 patients (21\%) only at the DTA. DTA, descending thoracic aorta.
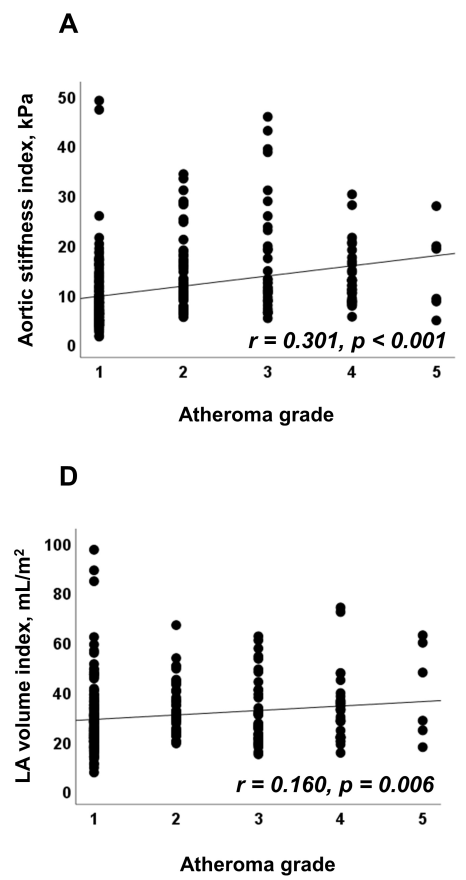

B



E



C

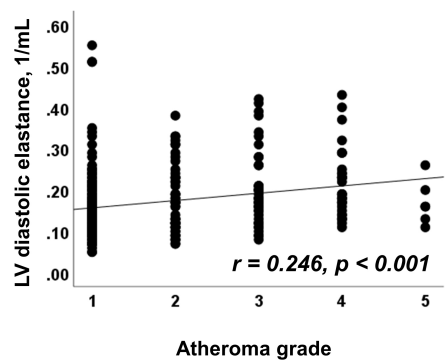

$\mathbf{F}$

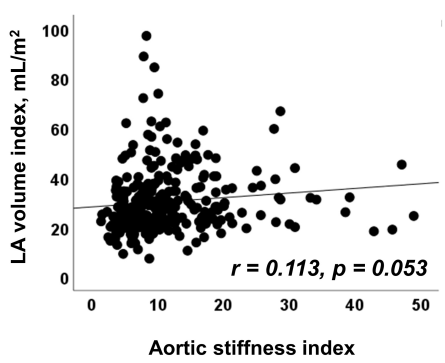

Fig. 2. Relationship between the grade of atheroma and aortic stiffness index, E/e', and LV diastolic elastance (A-C), and LA volume index (D-F) using univariate simple linear regression. The grade of atheroma is significantly related to aortic stiffness index (A), E/e' (B), and LV diastolic elastance (C). Among several aortic parameters, the atheroma grade (D) and aortic distensibility (E) are significantly relevant to LA volume index, but aortic stiffness index are not (F). LA, left atrial; LV, left ventricular. 
Total $(n=295)$

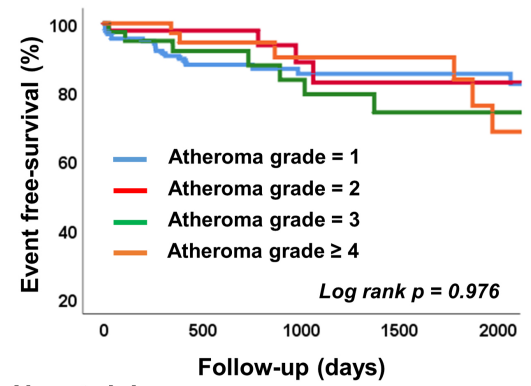

No. at risk
Men $(n=209)$

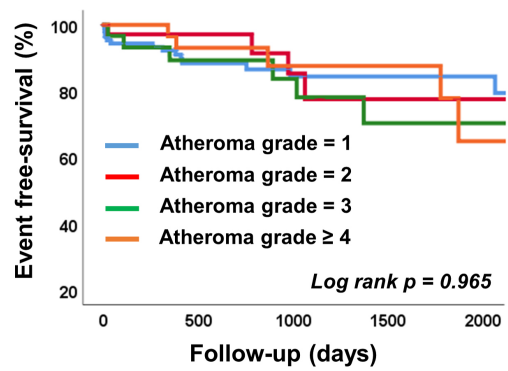

No. at risk
Women $(n=86)$



No. at risk

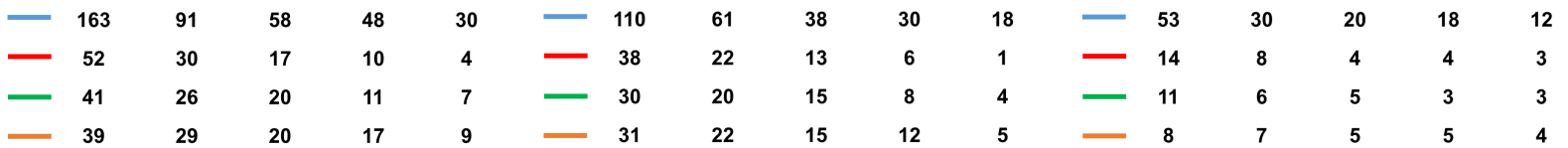

Fig. 3. Kaplan-Meier analysis for composite outcomes according to the grade of aortic atheroma as 1, 2, 3, and at least 4. There is no difference in clinical outcome ( $\log \operatorname{rank} p=0.976)$. The subgroups based on sex differences also show no difference in clinical outcomes (men, $\log$ rank $p=0.965$, women, $\log \operatorname{rank} p=0.951$ ).

$\mathrm{mL} / \mathrm{mmHg}, p=0.033$ ) compared with men. After TEE, the arterial stiffness index and distensibility index were not significantly different between both sexes.

\subsection{The association between aortic atheroma, aortic stiffness, and LV diastolic function}

The patients with any atheroma (higher than grade 1) had higher aortic stiffness index $(14.5 \pm 8.3 \mathrm{kPa}$ vs. $8.8 \pm$ $6.3 \mathrm{kPa}, p<0.001)$, E/e' $(11.9 \pm 3.6$ vs. $10.0 \pm 3.5, p<$ $0.001), \mathrm{LV}$ diastolic elastance $(0.19 \pm 0.081 / \mathrm{mL}$ vs. 0.16 $\pm 0.081 / \mathrm{mL}, p<0.001)$ and LA volume index $(32.7 \pm$ $12.2 \mathrm{~mL} / \mathrm{m}^{2}$ vs. $\left.28.3 \pm 13.1 \mathrm{~mL} / \mathrm{m}^{2}, p=0.003\right)$ than patients without any atheroma (grade 1$)$. The aortic atheroma grade was significantly related with aortic stiffness index $(\mathrm{r}$ $=0.301, p<0.001), \mathrm{E} / \mathrm{e}^{\prime}(\mathrm{r}=0.248, p<0.001)$, and LV diastolic elastance $(\mathrm{r}=0.246, p<0.001)$, and these correlations were concordant when the group was divided according to sex. Their relationships were more significant in women. The atheroma grade $(\mathrm{r}=0.144, p=0.038)$ and aortic distensibility $(\mathrm{r}=-0.136, p=0.049)$ in men and aortic stiffness index $(\mathrm{r}=0.302, p=0.005)$ and distensibility $(\mathrm{r}=$ $-0.274, p=0.011)$ in women were significantly correlated with LA volume index (Fig. 2 and Supplementary Table 2). After further adjustment for age, hypertension, diabetes, estimated glomerular filtration rate, LA volume index, and LV mass index, which were significantly related to diastolic function, the significance of atheroma grade was attenuated. However, when the analyses were performed for each sex, the atheroma grade was significantly and independently related to $\mathrm{E} / \mathrm{e}^{\prime}(\beta=0.181, p=0.032)$ in the female group, but not in the male group $(\beta=0.000, p=0.998)$. No aortic parameters were independently correlated with LA volume index after adjustment for other confounding factors (Table 3).

\subsection{Aortic atheroma and clinical outcomes}

During a mean follow-up of $2.9 \pm 2.6$ years, a total of 41 patients $(13.9 \%)$ experienced clinical outcomes. There was no difference in the incidence of clinical outcomes between both sexes. When the entire cohort was divided according to the grade of aortic atheroma as 1,2, 3, and at least 4 , there was no difference in clinical outcomes (log rank $p=0.976)$. The subgroups divided based on sex differences also showed no difference in clinical outcomes (men, $\log$ rank $p=0.965$, women, $\log$ rank $p=0.951$ ) (Fig. 3). In multivariate Cox regression analysis of the entire study population, there were no clinical variables independently associated with composite outcomes. In the subgroup of female patients, LA volume index was the only independent prognostic effect with a poor composite outcome (hazard ratio [HR]: 1.07, 95\% confidence interval [CI]: 1.02-1.12, $p=0.008)$; however, the other clinical variables were not (Table 4).

\section{Discussion}

The principal results of the study are as follows: (1) The presence and degree of aortic atheroma in ischemic stroke or TIA patients were significantly correlated with aortic stiffness index, E/e', LV diastolic elastance, and LA volume index. (2) The grade of aortic atheroma in women was independently related to E/e'. (3) The LA volume index, which reflects diastolic function, was independently associated with poor prognosis in patients, especially in women. These findings imply that aortic atheroma is a potential risk factor for HFpEF through ventricular-vascular interactions and should be managed appropriately to restrain the aggravation of heart failure. 
Table 3. Multiple linear regression analysis for $\mathrm{E} / \mathrm{e}^{\prime}$ and LA volume index with aortic parameters.

\begin{tabular}{|c|c|c|c|c|c|c|}
\hline & \multirow{2}{*}{\multicolumn{2}{|c|}{$\begin{array}{c}\text { Total } \\
(\mathrm{n}=295)\end{array}$}} & \multirow{2}{*}{\multicolumn{2}{|c|}{$\begin{array}{c}\text { Male } \\
(\mathrm{n}=209)\end{array}$}} & \multirow{2}{*}{\multicolumn{2}{|c|}{$\begin{array}{l}\text { Female } \\
(n=86)\end{array}$}} \\
\hline & & & & & & \\
\hline & $\beta$ & $p$ value & $\beta$ & $p$ value & $\beta$ & $p$ value \\
\hline \multicolumn{7}{|l|}{ For E/e' } \\
\hline Atheroma grade & 0.028 & 0.595 & 0.000 & 0.998 & 0.181 & 0.032 \\
\hline Aortic stiffness index & -0.004 & 0.945 & -0.050 & 0.459 & 0.128 & 0.181 \\
\hline Age & 0.304 & $<0.001$ & 0.221 & 0.007 & 0.330 & 0.027 \\
\hline Hypertension & 0.187 & 0.001 & 0.211 & 0.001 & 0.056 & 0.571 \\
\hline Diabetes mellitus & 0.144 & 0.004 & 0.163 & 0.006 & 0.175 & 0.049 \\
\hline eGFR, per $\mathrm{mL} / \mathrm{m}^{2}$ & 0.039 & 0.535 & -0.052 & 0.495 & 0.118 & 0.326 \\
\hline LA volume index, $\mathrm{mL} / \mathrm{m}^{2}$ & 0.200 & $<0.001$ & 0.210 & 0.001 & 0.204 & 0.036 \\
\hline LV mass index, $\mathrm{g} / \mathrm{m}^{2}$ & 0.150 & 0.005 & 0.200 & 0.002 & 0.130 & 0.199 \\
\hline \multicolumn{7}{|l|}{ For LA volume index } \\
\hline Atheroma grade & 0.030 & 0.624 & 0.032 & 0.673 & -0.023 & 0.811 \\
\hline Aortic stiffness index & -0.038 & 0.621 & -0.099 & 0.301 & 0.086 & 0.504 \\
\hline Aortic distensibility & -0.002 & 0.979 & -0.050 & 0.615 & 0.232 & 0.107 \\
\hline Age & 0.237 & 0.003 & 0.153 & 0.106 & 0.693 & $<0.001$ \\
\hline Hypertension & -0.031 & 0.619 & -0.046 & 0.543 & -0.077 & 0.502 \\
\hline Diabetes mellitus & 0.029 & 0.605 & 0.015 & 0.827 & 0.025 & 0.811 \\
\hline $\mathrm{eGFR}$, per $\mathrm{mL} / \mathrm{m}^{2}$ & -0.016 & 0.822 & -0.109 & 0.210 & 0.323 & 0.020 \\
\hline LV mass index, $\mathrm{g} / \mathrm{m}^{2}$ & 0.297 & $<0.001$ & 0.251 & $<0.001$ & 0.406 & $<0.001$ \\
\hline
\end{tabular}

Table 4. Multivariate Cox regression analysis for composite outcome.

\begin{tabular}{|c|c|c|c|c|c|c|c|c|c|}
\hline & \multicolumn{3}{|c|}{ Total } & \multicolumn{3}{|c|}{ Male } & \multicolumn{3}{|c|}{ Female } \\
\hline & \multicolumn{3}{|c|}{$(\mathrm{n}=295)$} & \multicolumn{3}{|c|}{$(\mathrm{n}=209)$} & \multicolumn{3}{|c|}{$(\mathrm{n}=86)$} \\
\hline & HR & $95 \% \mathrm{CI}$ & $p$ value & HR & $95 \% \mathrm{CI}$ & $p$ value & HR & $95 \% \mathrm{CI}$ & $p$ value \\
\hline Age & 1.00 & $0.97-1.03$ & 0.947 & 1.01 & $0.97-1.04$ & 0.796 & & & \\
\hline \multicolumn{10}{|l|}{ Hypertension } \\
\hline \multicolumn{10}{|l|}{ Diabetes mellitus } \\
\hline eGFR & 0.99 & $0.97-1.01$ & 0.418 & 0.99 & $0.96-1.01$ & 0.987 & & & \\
\hline \multicolumn{10}{|l|}{$\mathrm{E} / \mathrm{e}^{\prime}$} \\
\hline LA volume index & 1.01 & $0.99-1.03$ & 0.368 & & & & 1.07 & $1.02-1.12$ & 0.008 \\
\hline LV mass index & & & & & & & 1.01 & $0.98-1.04$ & 0.596 \\
\hline Aortic stiffness index & 1.02 & $0.98-1.07$ & 0.249 & 1.03 & $0.98-1.09$ & 0.194 & & & \\
\hline \multicolumn{10}{|l|}{ Atheroma grade } \\
\hline LV diastolic elastance & 38.34 & $0.82-1783.57$ & 0.063 & 76.73 & $0.42-14005.24$ & 0.102 & & & \\
\hline Effective arterial elastance & & & & 1.14 & $0.45-2.94$ & 0.780 & & & \\
\hline Total Arterial Compliance & & & & & & & & & \\
\hline
\end{tabular}

eGFR, estimated glomerular filtration rate; LA, left atrial; LV, left ventricular.

4.1 Aortic atheroma as an index of aortic stiffness and $L V$ diastolic functional impairment

Our study demonstrated that the grade of aortic atheroma was significantly associated with the index of aortic stiffness, E/e', and LA volume index, which reflects LV diastolic dysfunction. Although it is well known that aortic atheroma is associated with aortic stiffness and the degree of aortic stiffness is related to LV diastolic dysfunction
$[3,7]$, no correlations have been reported in previous studies that directly investigated the correlation between the grade of aortic atheroma and LV diastolic dysfunction. Till date, in patients with ischemic stroke, the presence and degree of aortic atheroma has been demonstrated to be a possible source of embolic events. However, our study revealed that the grade of aortic atheroma was significantly associated with diastolic dysfunction. Atherosclerotic plaque is 
not only a potential source of embolism in patients with ischemic stroke, but can also be considered as an afterload index that reflects arteriosclerosis. Therefore, treatment that reduces arterial stiffness through prohibition of the renin-angiotensin-aldosterone system as well as statin and antiplatelet treatment should be considered.

\subsection{Sex differences in the association between aortic atheroma and $L V$ diastolic dysfunction}

In our study, the grade of aortic atheroma was independently correlated with LV diastolic dysfunction, which was represented by E/e' in women $(\beta=0.181, p=0.032)$ but not in men. Various studies have demonstrated the association between aortic stiffness and diastolic dysfunction in women [9,13,17,18]. Shim et al. [9] demonstrated this relationship by using the central pulse pressure as an indices of aortic stiffness and revealed that the associations of central pulse pressure with e' velocity and E/e' were significant in female $(\mathrm{r}=0.38, p=0.001, \mathrm{r}=-0.36, p=0.001$, respectively). In another study, the index of aortic stiffness was used as aortic characteristic impedance $\left(Z_{c}\right)$, and the study demonstrated that higher $Z_{c}$ was associated with elevated $\mathrm{E} / \mathrm{A}$ ratio, grade of diastolic dysfunction, and index of ventricular-arterial coupling (effective arterial elastance/LV end-systolic elastance, Ea/Ees), especially in women [18]. Our study showed the relationship between atheroma grade, which was revealed to be associated with aortic stiffness index and diastolic dysfunction in women. Increased aortic stiffness causes increased LV afterload, impaired LV relaxation, reduced coronary perfusion, and induced subendocardial ischemia and fibrosis, leading to considerable LV diastolic dysfunction, especially in women [19,20]. This implies that the ventricular-vascular interaction is more prominent in women. Therefore, such an indicator of aortic stiffness is an important determinant to the pathophysiology of HFpEF. It is well known that prevalence of the HFpEF is more dominant in female, compared with male population. This is because the women have more susceptibility to cardiac dysfunction such as lower myocardial compliance, the inefficiency of cardiac metabolism, and vascular uncoupling than men [10]. In female patients, a small-sized LV chamber would be associated with abnormal LV chamber function and those conditions cause elevated LV filling pressure, which makes elevated E/e' [13]. Considering these underlying issues, solving the ventricular-vascular interaction is a significant treatment target of HFpEF [21]. Furthermore, it can be concluded that the reduction of LV afterload could be an important treatment strategy in female patients with ischemic stroke. This strategy is important because female gender is different from male in terms of the risk factors, subtype and severity of the ischemic stroke, and prognosis. In women, cardio-embolic infarction was more and lacunar infarction was less, and acute ischemic stroke was regarded as a severe comorbidity and had high mortality, compared with men [22].

\subsection{Prognostic value of aortic atheroma and distensibility} in ischemic stroke

In our study, a total cardiovascular event occurred in $14 \%$ of patients during the mid-term follow-up duration. Among the whole study population, the LA volume index, as an important index of diastolic function, was an independent poor prognosticator (HR: $1.07,95 \% \mathrm{CI}: 1.02-1.12, p$ $=0.008)$. However, the indices related to aortic atheroma or stiffness were not independently related to the LA volume index, regardless of sex differences. In a previous study, an increased LA volume index in HFpEF patients was associated with poor prognosis [23] and the results revealed that patients with a higher LA volume index $(>40$ $\mathrm{mL} / \mathrm{m}^{2}$ ) and higher maximal velocity of tricuspid regurgitation $(>3.1 \mathrm{~m} / \mathrm{s})$ had a considerably greater risk of composite outcomes $(p=0.014)$ than those with others. Our study also showed similar results, but the atheroma grade was not directly related to poor prognosis. Further prognostic research is therefore required. In a previous study, more than half of the patients with a silent lacunar stroke without any cognitive dysfunction showed minor alteration of neuropsychological function [24]. These changes were associated with the presence of the silent lacunar infarcts on the earlier status of small vessel disease. Based on these results of this research, in acute stroke patients with aortic atheroma, the future directions of this study will be able to determine whether the silent cerebral ischemia would be associated with further poorer functional outcomes.

\section{4 Limitations}

There are some considerable limitations in this study. First, this was a retrospective study and there were no available data on regular follow-up echocardiographic exams to distinguish the changes in vascular hemodynamics after the diagnosis of ischemic stroke. Second, there are limitations in the accurate measurement of the grade of aortic atheroma, especially in patients with atheroma located at the proximal aortic arch, which is difficult to access using TEE. Third, the mid-term follow-up duration was relatively short (mean 2.9 years), which might not be sufficient to evaluate the relationship between aortic atheroma and clinical prognosis. Forth, since not all patients diagnosed with ischemic stroke or TIA underwent TEE, there is a limitation in selection bias.

\section{Conclusions}

The severity of atheroma grade in the aorta was significantly and independently related to LV diastolic function, especially in women, and it supports previous studies regarding the higher sensitivity of ventricular-vascular interaction in women. This suggests that aortic atheroma is an index of arterial stiffness and a potential risk factor for HF$\mathrm{pEF}$ through ventricular-vascular interactions, especially in women. 


\section{Author contributions}

DYK and EYC designed this research study. YHJ, KYL, ISK, JYK, PKM, and YWY contributed to the data collection, analysis, and interpretation. BKL, BKH, and HMK contributed to statistical analysis. SJR, DYK, and EYC contributed to drafting the manuscript. All authors contributed to the manuscript. All authors read and approved the final manuscript.

\section{Ethics approval and consent to participate}

This study protocol was acknowledged by the Institutional Review Board of Gangnam Severance Hospital (3-2019-0241) the need for written informed consent was waived due to the study's retrospective design.

\section{Acknowledgment}

We thank to Minjee Kim to help us for data collection and administrative work for approving IRB process.

\section{Funding}

This work was supported by the Basic Science Research Program through the National Research Foundation of Korea (NRF) funded by the Ministry of Education (2019R1F1A1045911).

\section{Conflict of interest}

The authors declare no conflict of interest.

\section{Supplementary material}

Supplementary material associated with this article can be found, in the online version, at https://www.imrpre ss.com/journal/RCM/23/1/10.31083/j.rcm2301010.

\section{References}

[1] Mozaffarian D, Benjamin EJ, Go AS, Arnett DK, Blaha MJ, Cushman M, et al. Heart disease and stroke statistics-2016 update: a report from the American Heart Association. Circulation. 2016; 133: e38-e360.

[2] O'Rourke MF, Staessen JA, Vlachopoulos C, Duprez D, Plante GEE. Clinical applications of arterial stiffness; definitions and reference values. American Journal of Hypertension. 2002; 15: 426-444.

[3] Yambe M, Tomiyama H, Hirayama Y, Gulniza Z, Takata Y, Koji Y, et al. Arterial stiffening as a possible risk factor for both atherosclerosis and diastolic heart failure. Hypertension Research. 2004; 27: 625-631.

[4] Tunick PA, Kronzon I. Atheromas of the thoracic aorta: clinical and therapeutic update. Journal of the American College of Cardiology. 2000; 35: 545-554.

[5] Ferrari E, Vidal R, Chevallier T, Baudouy M. Atherosclerosis of the thoracic aorta and aortic debris as a marker of poor prognosis: benefit of oral anticoagulants. Journal of the American College of Cardiology. 1999; 33: 1317-1322.

[6] Sugioka K, Hozumi T, Sciacca RR, Miyake Y, Titova I, Gaspard $\mathrm{G}$, et al. Impact of aortic stiffness on ischemic stroke in elderly patients. Stroke. 2002; 33: 2077-2081.

[7] van Popele NM, Grobbee DE, Bots ML, Asmar R, Topouchian J, Reneman RS, et al. Association between arterial stiffness and atherosclerosis: the Rotterdam Study. Stroke. 2001; 32: 454 460.

[8] Bushnell CD, Chaturvedi S, Gage KR, Herson PS, Hurn PD, Jiménez MC, et al. Sex differences in stroke: Challenges and opportunities. Journal of Cerebral Blood Flow \& Metabolism. 2018; 38: 2179-2191.

[9] Shim CY, Park S, Choi D, Yang WI, Cho IJ, Choi EY, et al. Sex differences in central hemodynamics and their relationship to left ventricular diastolic function. Journal of the American College of Cardiology. 2011; 57: 1226-1233.

[10] Beale AL, Meyer PMD, Marwick TH, Lam CSP, Kaye DM. Sex differences in cardiovascular pathophysiology: why women are overrepresented in heart failure with preserved ejection fraction. Circulation. 2018; 138: 198-205.

[11] Lang RM, Badano LP, Mor-Avi V, Afilalo J, Armstrong A, Ernande $\mathrm{L}$, et al. Recommendations for cardiac chamber quantification by echocardiography in adults: an update from the American Society of Echocardiography and the European Association of Cardiovascular Imaging. European Heart Journal Cardiovascular Imaging. 2015; 16: 233-271.

[12] Ommen SR, Nishimura RA, Appleton CP, Miller FA, Oh JK, Redfield MM, et al. Clinical Utility of Doppler Echocardiography and Tissue Doppler Imaging in the Estimation of Left Ventricular Filling Pressures: a comparative simultaneous Dopplercatheterization study. Circulation. 2000; 102: 1788-1794.

[13] Ha JW, Lee HC, Park S, Choi E, Seo H, Shim C, et al. GenderRelated Difference in Left Ventricular Diastolic Elastance during Exercise in Patients with Diabetes Mellitus. Circulation Journal. 2008; 72: 1443-1448.

[14] Pujadas R, Arboix A, Anguera N, Rafel J, Sagués F, Casañas R. Mitral annular calcification as a marker of complex aortic atheroma in patients with stroke of uncertain etiology. Echocardiography. 2008; 25: 124-132.

[15] Goldstein SA, Evangelista A, Abbara S, Arai A, Asch FM, Badano LP, et al. Multimodality Imaging of Diseases of the Thoracic Aorta in Adults: from the American Society of Echocardiography and the European Association of Cardiovascular Imaging: endorsed by the society of cardiovascular computed tomography and society for cardiova. Journal of the American Society of Echocardiography. 2015; 28: 119-182.

[16] Rerkpattanapipat P, Hundley WG, Link KM, Brubaker PH, Hamilton CA, Darty SN, et al. Relation of aortic distensibility determined by magnetic resonance imaging in patients $\geq 60$ years of age to systolic heart failure and exercise capacity. The American Journal of Cardiology. 2002; 90: 1221-1225.

[17] Her AY, Kim JY, Choi EY, Kim SA, Jae RS, Shim CY, et al. Value of Ventricular Stiffness Index and Ventriculoarterial Interaction in Patients with Nonischemic Dilated Cardiomyopathy. Circulation Journal. 2009; 73: 1683-1690.

[18] Coutinho T, Borlaug BA, Pellikka PA, Turner ST, Kullo IJ. Sex differences in arterial stiffness and ventricular-arterial interactions. Journal of the American College of Cardiology. 2013; 61: 96-103.

[19] Park S, Ha JW, Shim CY, Choi E, Kim J, Ahn J, et al. Genderrelated difference in arterial elastance during exercise in patients with hypertension. Hypertension. 2008; 51: 1163-1169.

[20] Shim CY, Hong GR, Ha JW. Ventricular Stiffness and Ventricular-Arterial Coupling in Heart Failure: what is it, how to Assess, and why? Heart Failure Clinics. 2019; 15: 267-274.

[21] Shim CY. Heart failure with preserved ejection fraction: the major unmet need in cardiology. Korean Circulation Journal. 2020; 50: 1051-1061.

[22] Arboix A, Cartanyà A, Lowak M, García-Eroles L, Parra O, Oliveres $\mathrm{M}$, et al. Gender differences and woman-specific trends in acute stroke: results from a hospital-based registry (19862009). Clinical Neurology and Neurosurgery. 2014; 127: 19-24. 
[23] Donal E, Lund LH, Oger E, Bosseau C, Reynaud A, Hage C, et al. Importance of combined left atrial size and estimated pulmonary pressure for clinical outcome in patients presenting with heart failure with preserved ejection fraction. European Heart Journal Cardiovascular Imaging. 2017; 18: 629-635.
[24] Blanco-Rojas L, Arboix A, Canovas D, Grau-Olivares M, Oliva Morera JC, Parra O. Cognitive profile in patients with a first-ever lacunar infarct with and without silent lacunes: a comparative study. BMC Neurology. 2013; 13: 203. 